\title{
Convolutional neural networks for segmentation and object detection of human semen
}

\author{
Malte S. Nissen ${ }^{1,2,3,4}$, Oswin Krause ${ }^{1}$, Kristian Almstrup ${ }^{2,3}$, Søren Kjærulff ${ }^{4}$, \\ Torben T. Nielsen ${ }^{4}$, and Mads Nielsen ${ }^{1}$ \\ 1 Department of Computer Science, University of Copenhagen, Denmark \\ nissen@di.ku.dk \\ 2 Department of Growth and Reproduction, Rigshospitalet, University of \\ Copenhagen, Denmark \\ 3 International Center for Research and Research Training in Endocrine Disruption \\ of Male Reproduction and Child Health (EDMaRC), Rigshospitalet, University of \\ Copenhagen, Denmark \\ ${ }^{4}$ ChemoMetec A/S, Allerød, Denmark
}

\begin{abstract}
We compare a set of convolutional neural network (CNN) architectures for the task of segmenting and detecting human sperm cells in an image taken from a semen sample. In contrast to previous work, samples are not stained or washed to allow for full sperm quality analysis, making analysis harder due to clutter. Our results indicate that training on full images is superior to training on patches when class-skew is properly handled. Full image training including up-sampling during training proves to be beneficial in deep CNNs for pixel wise accuracy and detection performance. Predicted sperm cells are found by using connected components on the CNN predictions. We investigate optimization of a threshold parameter on the size of detected components. Our best network achieves $93.87 \%$ precision and $91.89 \%$ recall on our test dataset after thresholding outperforming a classical image analysis approach.
\end{abstract}

Keywords: deep learning, segmentation, convolutional neural networks, human sperm, fertility examination

\section{Introduction}

Sperm Quality Analysis (SQA) involves measuring concentration, morphology, and motility [13] of sperm cells. For the application to animal sperm cells, there exist a number of commercial Computer-Aided Sperm Analysis (CASA) systems, such as the Hamilton-Thorne IVOS-II and CEROS-II $]^{5}$ and the Sperm Class Analyzer 6

Human semen samples have a significantly lower quality of sperm cells compared to most animals [7, which increases the accuracy demand on the analysis.

5 http://www.hamiltonthorne.com/

6 http://www.micropticsl.com/products/sperm-class-analyzer-casa-system/ 


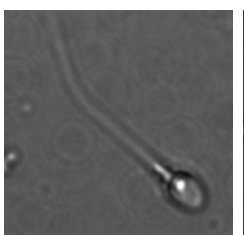

(a)

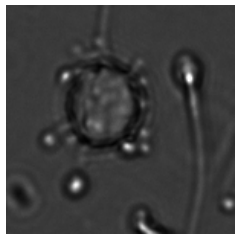

(f)

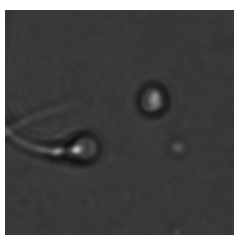

(b)

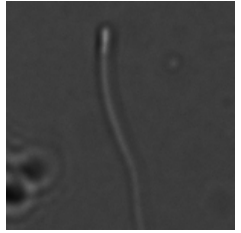

$(\mathrm{g})$

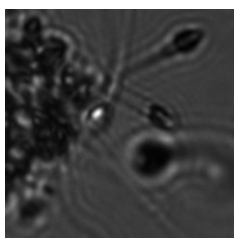

(c)

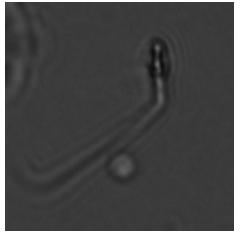

(h)

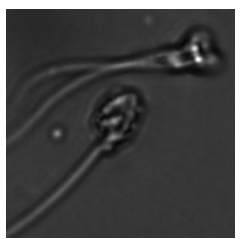

(d)

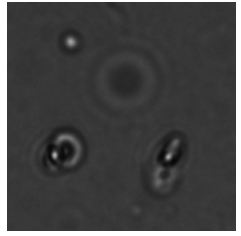

(i)

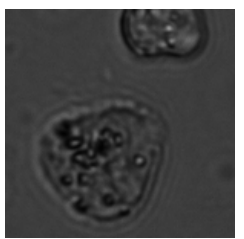

(e)

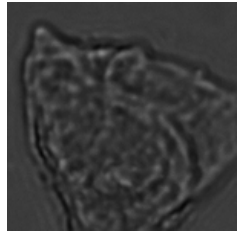

(j)

Fig. 1: Examples of debris, variations, and morphological abnormalities: normal sperm cell (a. b), aggregated cells out of focus (c), agglutinated cells (d), round cells (e, f), headless sperm (g), sperm head seen from the side or morphologically abnormal (h, i), circular tails (i), and other types of artifacts and debris (b, f] j).

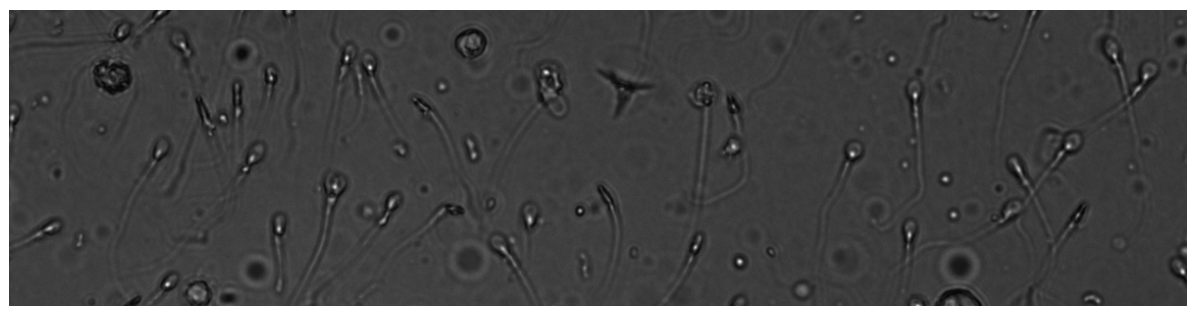

Fig. 2: $1200 \times 300$ pixel cut-out of image from the dataset

Moreover, human semen is often cluttered with debris and cells other than normal mature sperms. Fig. 1 shows examples of typical debris, variations and morphological abnormalities of human sperm samples. Fig. 2 shows a section of a typical image.

In practice, staining and smearing are often used for preparation of samples to highlight specific properties of the cells $[1|2| 3|4| 10$, but the sample needs to be in its natural form for motility estimation. This article focuses on the first step of SQA, image segmentation and detection of non-stained human sperm cells as analyzed by Ghasemian et al. (2015) [4] and Hidayahtullah et al. (2014) 6]. These algorithms apply classical image analysis techniques to solve the problem. To our knowledge no deep learning techniques have been applied yet.

Our approach focuses on deep convolutional neural networks (CNN) to segment the sperm cells in the image. There are three main challenges in this approach: Firstly, every pooling layer in a CNN reduces resolution by at least $50 \%$; after three layers of pooling, every pixel of the result encodes the information of 


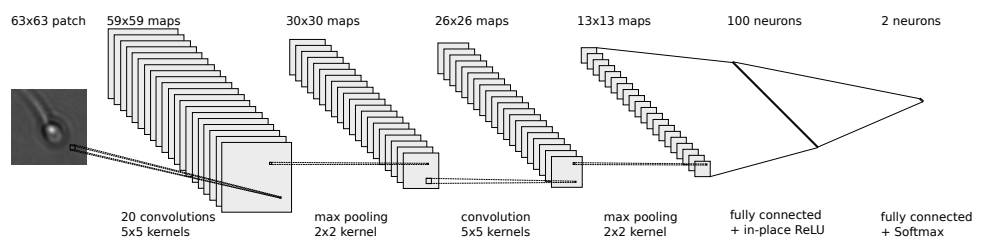

Fig. 3: Illustration of the 2-conv CNN

an $8 \times 8$ area of the original image. Secondly, CNNs are often trained on image patches, however there is a huge class imbalance between background and sperm pixels, where sperm pixels are significantly harder to detect. Lastly, we need to cluster the segmentations to objects. Imperfect predictions of the networks often lead to spurious detections, which need to be removed. One way to do this is to use thresholding on the size of clusters, leading to an arbitrary threshold parameter. This parameter needs to be chosen carefully.

We investigate possible solutions to these challenges. While using max-pooling layers is possible without reducing resolution [5], an exponential amount of time in the number of pooling layers is required. This makes it infeasible in practice as the results have to be computed quickly enough to allow video analysis. We follow Long et al. (2015) 9] and investigate up-sampling on the output of the CNN during training and testing. Ronneberger et al. (2015) [11] proposed a more complex architecture, which we disregard since predictions would be too slow for our application. Further, we compare training on image patches with training on the full images, where class-labels are re-weighted to correct the class-skew.

For comparison we implemented the sperm head detection method proposed by Ghasemian et al. (2015) 4]. This method has a similar threshold parameter as our method which has to be adapted for a fair comparison. For this, we propose a way to adapt the thresholding parameters using the product of precision and recall on the final detections.

The paper is organized as follows: Section 2 describes the dataset and the CNN architectures used. Experiments are described in Section 3. Results are given in Section 4 and discussed in Section 5 . Finally, we conclude in Section 6.

\section{Method}

Dataset. We have constructed a dataset of 765 grayscale images of 35 independent sperm samples. The 35 samples were individually diluted using a solution of Bicarbonate-Formalin (as devised by WHO [13]) to get an appropriate amount of cells in each image (between 2 and 290 sperm cells) and to fixate them. Fixation facilitates sedimentation of the cells to the bottom of the counting chamber, ensuring that all cells are roughly in the same focal plane. In order to have cells both in and out of focus, reflecting the optical variation, Z-stacks of images were acquired. The images were acquired using an image cytometer with $20 \times$ optical magnification and a resolution of $1920 \times 1440$ pixels $(0.2 \mu \mathrm{m} /$ pixel $)$. The 
image intensities have been quantized from 14- to 8-bit images. In each image the intensities where normalized to lie between zero and one.

The images were annotated by experts and registered into two classes: background and sperm cells. Round cells form an important part of the background and were therefore also annotated. The tip of the head and the neck point was registered for each sperm cell while the circumference was annotated for each round cell. Pixel-segmentation ground truths are generated by creating an ellipse at the center of each sperm cell head with radius $r_{1}=\frac{1}{4} l_{\text {cell }}$ and $r_{2}=\frac{2}{3} r_{1}$ where $l_{\text {cell }}$ is the length of the cell head.

We split the samples into $70 \%$ train and $30 \%$ test data based on stratified sampling on the average number of sperm cells in the full images of each sample. This ensures that images from the same sample are part of the same split as they contain correlated data. Hence, one sample being part of testing data is never represented in the training data.

From the training dataset we generated an additional dataset of extracted patches from the images using the annotated classes. This patch dataset contains $63 \times 63$ pixel patches which are labelled by their ground truth in the center pixel. The size of the patches is chosen to allow the entire head, which is typically 25 pixels long, and a small part of the tail to be included. From each image, we extract up to 3,000 patches, split into $40 \%$ sperm cells, $40 \%$ background and $20 \%$ round cells. The numbers were chosen to cover the variety of debris in the background class (round cells contribute a lot to the variability of the background). Random rotation and flipping is applied before extracting each patch. Table 1 shows statistics for the resulting datasets. Note that the dataset contains a total of 38,708 sperm cells of which 23,997 are included in the train set and 14,711 are included in the test set.

Networks. We define seven networks to test against each other. The first network is called 2-conv. It is defined for input patches and illustrated in Fig. 3. It is a standard CNN with two convolutional, ReLU and max-pooling layers followed by two fully connected layers separated by another ReLu layer and including $50 \%$ dropout during training. The network 3-conv is obtained by adding an additional set of convolution, ReLU, and max-pooling layers. The networks are defined with receptive fields of size $63 \times 63$ using 20 filters in each of their convolution layers and 100 filters in their fully convolutional layer.

For prediction on the full images, the fully connected layers are substituted with fully convolutional layers as described by Long et al. (2015) 9] to allow for faster computation. As each max-pooling layer divides the spatial resolution of the output by a factor of 2 in each dimension, we further perform bilinear upscaling of the network output probabilities to obain a pixel-wise segmentation.

To compare whether training on full images is beneficial compared to patchbased training, we define the architectures 2-conv-full and 3-conv-full, which have the same structure as 2-conv and 3-conv in the prediction phase and are trained on full images with the final up-sampling removed. Finally, the architectures 2-conv-full-up and 3-conv-full-up also incorporate the bilinear up-sampling into the training process. The networks trained on full images use a receptive field of 


\begin{tabular}{lrrr}
\hline Statistic & Train & Test & Total \\
\hline Images & 540 & 225 & 765 \\
Sperm cells & 23,997 & 14,711 & 38,708 \\
Patches & $1,424,341$ & 601,290 & $2,025,631$ \\
\hline
\end{tabular}

Table 1: Data statistics

\begin{tabular}{lccc}
\hline Method & $m_{I U}$ & Threshold & $m_{\text {pred }}(\mathrm{s})$ \\
\hline 2-conv & 0.6658 & 200 & 0.145 \\
2-conv-full & 0.7080 & 200 & 0.143 \\
2-conv-full-up & 0.6805 & 250 & 0.143 \\
3-conv & 0.6556 & 200 & 0.119 \\
3-conv-full & 0.6497 & 150 & 0.119 \\
3-conv-full-up & 0.6661 & 300 & $\mathbf{0 . 1 1 6}$ \\
3-conv-full-up-inc & $\mathbf{0 . 7 3 8 7}$ & 150 & 0.364 \\
baseline [4] & 0.5679 & 400 & -
\end{tabular}

Table 2: Experiment results $m_{I U}$, threshold, and $m_{\text {pred }}$ for all eight methods

size $64 \times 64$ and the same number of filter 7 . We further add a network 3-convfull-up-inc with the the same receptive field size but with 64, 128, and 256 filters in the convolution layers and 1024 filters in the fully convolutional layer. We omit the network 2-conv-full-up-inc due to limitations in the framework used.

When testing the networks, we perform post-processing of the full size output probabilities in two steps: Firstly, we choose the most probable class as output for each pixel. Secondly, we cluster pixel-wise segmentation to objects by computing the 8-neighbourhood connected components and removing components smaller than a threshold $t$. The value of this threshold is found in section 4

\section{Experiments}

The 2-conv and 3-conv architectures have been trained on the patch dataset and tested on the full image dataset, whereas all other networks have been trained and tested on the full image dataset. The outputs of 2-conv-full and 3-conv-full are smaller than the label masks of the full images. We therefore downsample the label masks by factors 4 and 8 respectively. This is done by taking every 4 th or 8th pixel corresponding to the center of the receptive field of the output.

All networks are trained by optimizing the cross-entropy between the predicted and ground truth label. To compensate for the class skew in the full images during training we re-weight the classes according to their distribution. The weight $w_{i}$ of class $i$ is defined as $w_{i}=\frac{1}{n_{i} \sum_{j} \frac{1}{n_{j}}}$ where $n_{i}$ is the number of

7 The difference comes from the fact that it is easier to define a center-pixel in $63 \times 63$ receptive fields 
pixels belonging to class $i$. Omitting the re-weighting led to far inferior results classifying everything as background.

The architectures have been trained for 200 epochs using the Adam solver [12] with mini-batches of 256 patches or 1 full image (1920 - 1440 "samples"). For training we chose learning rate $\alpha=0.001$, moment $1 \beta_{1}=0.9$, moment 2 $\beta_{2}=0.999$, and $\epsilon=10^{-8}$. We implemented the networks using Caffe [8], and the experiments have been carried out using a single Titan X GPU.

The baseline method [4] consists of three major steps: Noise reduction, object region detection, and sperm head localization. The method assumes that all sufficiently large object regions are sperm cells and therefore filters out all object regions smaller than a chosen threshold. This threshold is crucial for the performance of the algorithm and needs to be chosen carefully.

On an object level we are interested in finding each sperm cell. For this purpose we use the two measures precision $=\frac{T P}{T P+F P}$ and recall $=\frac{T P}{T P+F N}$, where $T P$ is the number of true positives, $F P$ is the number of false positives and $F N$ is the number of false negatives. A predicted sperm cell is categorized as TP if it covers more than half the area of a ground truth sperm cell. Each predicted cell can only count as one positive, i.e. a predicted cell covering more than half the area of two sperm cells counts as one true positive and one false negative. We evaluate precision and recall for multiple thresholds on the training data to get a precision-recall (PR) curve for every method. We choose the threshold value that maximizes the product between precision and recall.

Mean intersection over union (mean IU) $m_{I U}$ is used to quantify the pixelwise segmentation performance as described by Long et al. (2015) [9]:

$$
m_{I U}=\frac{1}{2} \sum_{i}\left(\frac{p_{i i}}{\sum_{j}\left(p_{i j}+p_{j i}\right)-p_{i i}}\right)
$$

Where $p_{i j}$ is the number of occurences of class $i$ predicted as class $j$. We have chosen this measure since it is invariant to the aforementioned class skew.

Finally, fast computations is one of the requirements for automatic SQA. We therefore record the execution time of computing a prediction and object removal on all 765 full images and compute the mean execution time $m_{\text {pred }}$ per image. Our baseline method implementation is not as optimized as our networks and therefore we omit the results. 


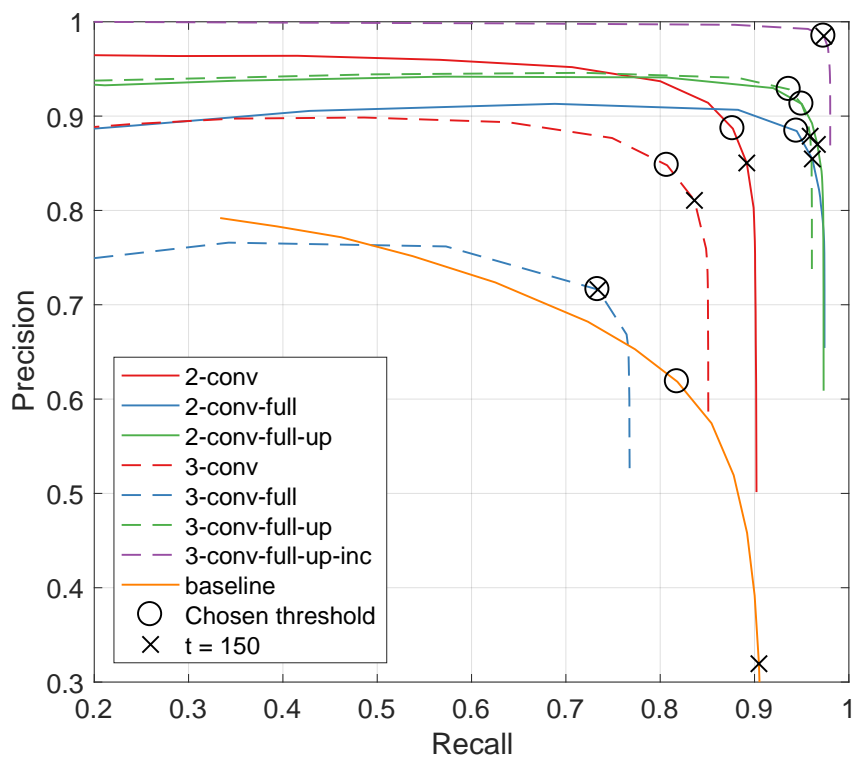

(a)

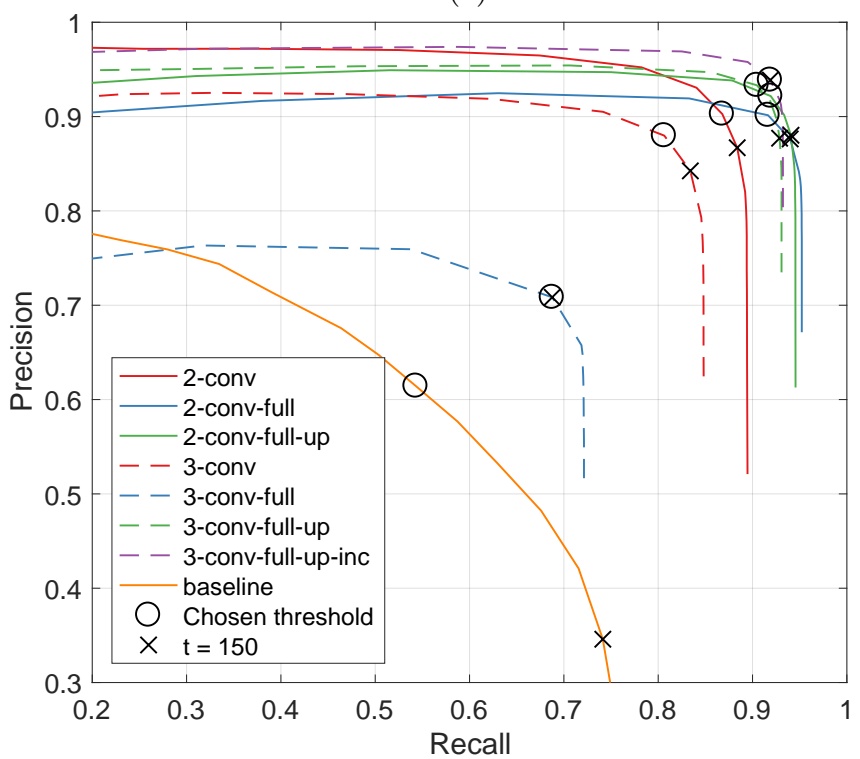

(b)

Fig. 4: Precision-recall graphs for (a) train and (b) test for all networks. 2-conv networks are plotted using fully-drawn lines and 3-conv networks using dashed lines. Same colours of lines indicate same parameters. The black circles indicate the point on each graph that corresponds to the threshold $t$ reported in Table 2 . while the black crosses indicate the points for $t=150$. 


\section{Results}

Results of the mean IU $m_{I U}$, thresholds found by maximizing the product between precision and recall on training data, and mean execution time $m_{\text {pred }}$ for each method are given in Table 2

Generally when considering $m_{I U}$, the networks trained on full images with up-sampling perform better than the networks trained on patches. All networks perform better than the baseline method. Training on full images without upsampling leads to better results for 2-conv-full but worse for 3-conv-full. The 2 -conv networks perform better than their 3-conv equivalents. The network 3conv-full-up-inc performs best, but it also has a considerably higher execution time $m_{\text {pred }}=0.364$ than the other methods spanning the range of $0.116-0.145$ seconds per image.

The results for the object detection are given in Fig. 4. The figure shows the precision-recall graphs for (a) train and (b) test for all methods. The graphs have ends due to the smallest and largest thresholds considered (0-1,000). We plot 2conv networks using fully-drawn lines and 3-conv networks using dashed lines. Same colours of lines indicate same parameters. The black circles indicate the point on each graph that corresponds to the threshold reported in Table2, while the black crosses indicate the points for a threshold of 150 . The baseline performs considerably different on the train and test set even though there is no training involved apart from the choice of threshold. It performs considerably worse than our networks except 3-conv-full. The best method is 3-conv-full-up-inc having $93.87 \%$ precision and $91.89 \%$ recall on the test set using threshold 150 . While some overfitting can be seen between training and test, it still outperforms the other methods.

\section{Discussion}

Our results show that using neural networks is beneficial compared to the classical approach. The large difference in baseline performance indicate that there is a large variation between samples. We believe that we have captured the variation of a sperm cell in our train and test sets, but we have not captured all possible combinations of cells in an entire image. Given our limited number of individual samples, there are some cell concentration differences. The baseline performance difference is likely caused by these cell concentration differences. Our networks are not affected by these differences except to the degree expected from overfitting. All networks except 3-conv-full-up-inc perform almost the same on train and test data whereas 3-conv-full-up-inc is showing clear signs of overfitting. This indicates that our networks are sufficiently complex to cover the variation of the data and that even larger networks are unlikely to generalize better. As we have not used the test set for model selection, we can expect the performance on the test set to be close to the true performance.

Up-sampling has different effects on mean IU and object detection. For mean IU detecting object boundaries is important. As up-sampling is equivalent to 
blurring it is not beneficial for mean IU when the model is already able to accurately describe the shape of the objects. This can be seen in the difference in its effect on networks with two and three max-pooling layers. We hypothesize that training using up-sampling gives us true predictions with cluster areas closer to the true size of sperm cells. This makes it easier to distinguish sperm cells from a specific type of debris (Fig. $1 \mathrm{~b} \&$ i) easily mistaken for the head of a sperm cell but having a slightly smaller area.

When omitting up-sampling, there is no general tendency when comparing patch-based and full-image training. For 2-conv networks, full-image training seems to profit from the increased variation in the data while patch-based training profits from the weighting of round cells in the background. This can be seen by the differences in precision and recall for the two methods in Fig. 4 .

When we compare the PR-curves, we see that the choice of a fixed threshold can be misleading. It turns out that the ranking of the networks can change depending on the choice of it. However, the chosen thresholds on the training set lead to consistent rankings on the test set in our case. Introducing the threshold and optimizing it leads to far superior results for all networks compared to choosing an arbitrary value. The obtained precision and recall seems reasonable for the purpose of identifying sperm cells in a semen sample, however it needs clinical testing for verification of its performance in practice.

\section{Conclusion}

In this paper, we have used deep convolutional neural networks for the task of sperm cell segmentation and object detection. In this task, we are constrained by the computation time as well as the accuracy demands, which make it harder to train networks with many pooling layers. To mitigate both problems we explored the use of full image training and up-sampling of the network outputs in order to increase performance. We specifically investigated thresholding on the size of detected components. Choosing the product of precision and recall leads to a robust estimate of threshold parameter. For deeper networks, up-sampling appears necessary to achieve good segmentation and object detection performance. The same does not necessarily hold for more shallow networks.

Our method outperformed a classical image analysis method which can be considered state-of-the-art. Overall the system sensitivity and precision are sufficiently high to be valuable for human sperm analysis systems.

\section{Acknowledgements}

This work is partly funded by the Innovation Fund Denmark (IFD) under File No. 4135-00169B. We would like to thank Department of Growth and Reproduction, Rigshospitalet, Denmark, for helping with annotation of our data. 


\section{References}

1. Bijar, A., Pe, A., Mikaeili, M. and others: Fully automatic identification and discrimination of sperm's parts in microscopic images of stained human semen smear. Scientific Research Publishing (2012)

2. Carrillo, H., Villarreal, J., Sotaquira, M., Goelkel, M.A., Gutierrez, R.: A computer aided tool for the assessment of human sperm morphology. Proceedings of the 7th IEEE International Conference on Bioinformatics and Bioengineering. 1152-1157 (2007)

3. Chang, V., Saavedra, J.M., Castañeda, V., Sarabia, L., Hitschfeld, N., Härtel, Steffen: Gold-standard and improved framework for sperm head segmentation. Computer Methods and Programs in Biomedicine. 117, 2, 225-237 (2014)

4. Ghasemian, F., Mirroshandel, S.A., Monji-Azad, S., Azarnia, M., Zahiri, Z.: An efficient method for automatic morphological abnormality detection from human sperm images. Computer Methods and Programs in Biomedicine. 122, 3, 409-420 (2015)

5. Giusti, A., Cireşan, D.C., Masci, J., Gambardella, L.M., Schmidhuber, J.: Fast image scanning with deep max-pooling convolutional neural networks. arXiv preprint arXiv:1302.1700 (2013)

6. Hidayatullah, P., Zuhdi, M.: Automatic sperms counting using adaptive local threshold and ellipse detection. International Conference on Information Technology Systems and Innovation (ICITSI). 56-61 (2014)

7. van der Horst, G., Mortimer, S. T, Mortimer, D.: The future of computer-aided sperm analysis. Asian Journal of Andrology. 17, 4 (2015)

8. Jia, Y., Shelhamer, E., Donahue, J., Karayev, S., Long, J., Girshick, R., Guadarrama, S., Darrell, T.: Caffe: Convolutional Architecture for Fast Feature Embedding. arXiv preprint arXiv:1408.5093 (2014)

9. Long, J., Shelhamer, E., Darrell, T.: Fully convolutional networks for semantic segmentation. Proceedings of the IEEE Conference on Computer Vision and Pattern Recognition. 3431-3440 (2015)

10. Medina-Rodríguez, R., Guzmán-Masías, L., Alatrista-Salas, H., Beltrán-Castañón, C.: Sperm cells segmentation in micrographic images through lambertian reflectance model. Springer. Computer Analysis of Images and Patterns. 664-674 (2015)

11. Ronneberger, O., Fischer, P., Brox, T.: U-net: Convolutional networks for biomedical image segmentation. Springer. International Conference on Medical Image Computing and Computer-Assisted Intervention. 234-241 (2015)

12. Kingma, D. P., Ba, J.: Adam: A method for stochastic optimization. arXiv preprint arXiv:1412.6980 (2014)

13. World Health Organization and others: WHO laboratory manual for the examination and processing of human semen. Geneva: World Health Organization (2010) 